\title{
Cellular Automata in Topology Optimization of Continuum Structures
}

\author{
E. Sanaei $^{1 *}$, M. Babaei ${ }^{2}$ \\ ${ }^{1 *}$ Department of Civil Engineering, Iran University of Science and Technology, IRAN \\ ${ }^{2}$ Department of Civil Engineering, Iran University of Science and Technology, IRAN \\ *Corresponding Author: e-mail: sanaei@iust.ac.ir, Tel +98-21-77451501, Fax +98-21-77451502
}

\begin{abstract}
In this paper, an optimization algorithm based on cellular automata (CA) is developed for topology optimization of continuum structures with shear and flexural behavior. The design domain is divided into small triangle elements and each cell is considered as a finite element. The stress analysis is performed by the Constant Strain Triangles (CST) finite elements method. The thicknesses of the individual cells are taken as the design variables, while the weight of the structure and the ratio of the Von Mises equivalent stress to the yield stress in each cell are considered as the two objective functions to minimize. Using the weighted sum method, the multi-objective optimization problem (MOOP) is converted to the single-objective optimization problem (SOOP) and then the optimization problem is solved by the developed method. The paper reports the results of several design experiments, comparing with the existing reported results.
\end{abstract}

Keywords: Continuum Structures, Cellular Automaton, Topology Optimization, Finite Element.

\section{Introduction}

In this competitive world, lack of the resources and the need for efficient structures create challenges for engineers to find costeffective and efficient solutions and designs. Skill, intuition, and experience of the designers can directly affect the designs. The design of complex and huge structures requires data processing and a large number of calculations. Computer-aided design optimization (CADO), however, has been developed during the last decades. The engineering design and optimization processes benefit vastly from the revolution of calculation using computers. The optimization methods, in the literature, are classified into two different categories; optimality criteria (indirect) methods and search (direct) methods.

Optimality criteria are conditions that must be satisfied by a function at its minimum point. Many mathematical (or deterministic) methods and stochastic (or probabilistic) methods are introduced, developed and applied for the optimization of structures, in the literature.

Cellular automaton (CA) was introduced by von Neumann (1966) and Ulam (1952), and it has been considered as a discrete simulation scheme in the last five decades. In general, in this method, cells are considered as similar squares or other shapes, and values of each cell in a special time-step are updated using local rules, regarding the status of the cell and its neighbor cells in the previous time-step. Kita and Toyoda (2000) presented a scheme for optimization of structures by using the concept of a CA, dividing the design domain into small square cells. Kita and Toyoda applied the proposed scheme to a two-dimensional elastic problem to confirm its validity. Since these rules are to introduce existing relationships between adjacent and neighboring cells, it is not necessary to know the general rules governing the issue. Thus, CA is an appropriate method for problems where the accurate information of the general relations is not available.

CA has been used for simulating a variety of problems such as fluid flow and transportation traffic; however, the main idea of applying this method in structural shape optimization for the first time was proposed by Inou et al (1994). The basic idea as described by Inou et al. $(1994,1998)$ is to divide the design domain into small cells and then to obtain von Mises equivalent stress distribution in the cells using the finite element method. Then the amount of stress in each cell is updated using the values of stress in neighboring cells and applying local rules. In this method, Young's modulus is considered as a design variable and it is modified in every stage such that the stress of each cell becomes equal to the amount of stress of the cell obtained in the stage. 
Thus by eliminating cells with relatively small Young's modulus, the goal of shape and geometry optimization of structures are simultaneously implemented.

The local rule applied in these studies is nonlinear relationship between the cell stresses, and the Young modulus has to be considered. The numerical experience shows that there is no reliable connection between this method and mathematical optimization problem. Since the stresses in each cell are updated individually during the optimization process, it is not possible to apply suitable stress constraints.

On the other hand Xie et al. (1993, 1994, and 1997) introduced an evolutionary structural optimization (ESO). In this scheme, the first base value is determined. After analysis using the finite element method, cells with smaller stress than the base amount are removed. In their recent studies, the ESO method of evolutionary structural optimization has been generalized. In this scheme two base values are introduced. Thus, while some cells using the first criterion are removed, another group of cells with regard to other criteria are added. However, the physical concepts of these base quantities are not specified and therefore they should be determined by previous numerical results or previous research experiments. To overcome the above problems, the following algorithm is presented and used. First, the design domain area is divided into small triangle cells and thickness of each cell is considered as a design variable, as illustrated in figure 1.

In the next step, the whole problem of structural optimization is converted to the optimization of each cell using CA constraint condition. Formulation of this method does not involve entering new parameters whose physical nature is not clear, which is considered an advantage of using this method.

In this paper, a new algorithm is presented and generalized to continuum structures. Two type of design experiments are tested using the proposed algorithm, including deep and simple beams. The remaining sections of this paper are organized as follows. Section 2 includes the review of CA. The developed finite element routine is described in Section 3. Section 4 describes the problem formulation for optimum design. In Section 5, the execution process and the proposed algorithm are given. Section 6 contains several design examples which are classified into two types of beams, deep and simple, and Section 7 concludes the article.

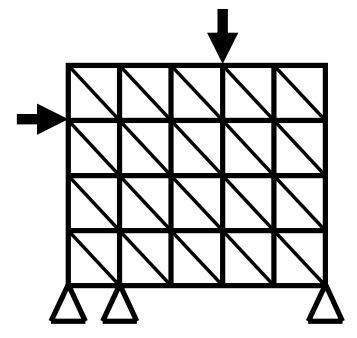

Figure 1. Design domain

\section{Cellular Automata}

CA is a mathematical model for systems in which many simple components for complex patterns can work together. CA is made up of a regular network, where each cell can take different amounts. The cells of CA at each step of implementation are updated simultaneously using a local rule in which the value of each cell is determined based on the values of neighboring cells. CA could be divided into various categories. For example, based on the dimension of network criteria, CA will be divided into one-dimensional, two-dimensional, or multi-dimensional. CA based on the amount of each cell is divided into two-value CA and multi-value.

CA based on the network neighbors can be divided into two categories, as CA with periodic boundary and non-periodic boundary. The most famous neighbors in the two-dimensional CA model are known as the Moore neighborhood and Von Neumann, as shown in figure 2(a, b). In this paper, a new neighborhood is developed based on triangular shape. The design domain is divided into triangles with three-node cells. A variety of neighborhoods can be considered for cells. However, here, only the cells that are in common ridge are selected as neighbors, as illustrated in figure 2(c).

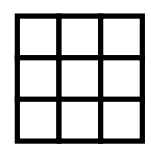

(a)

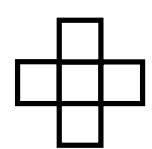

(b)

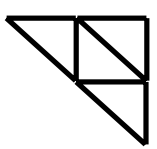

(c)

Figure 2. The popular neighbors in CA. (a) Moore, (b) von Neumann, (c) Triangular 
All of these cells are considered as independent components through the finite element analysis and stress distribution in each cell is determined. Usually, in the simulation process using CA, value of cells is considered as limited and a finite amount. However, in this paper, these values are considered as continuous quantities. The values of each cell in each step are determined based on the status of the cell and its neighbor cells in the previous step, using the appropriate local rule. Figure 2(c) shows the neighbor cells of the triangular elements. For the boundary cells or the cells located in the sides, only the adjacent cells are considered as neighboring cells.

CA is definitely a new comer to the field of structural analysis and design. Nevertheless, a number of methods that appear in the structural optimization literature have a basic structure reminiscent of CA algorithms. These methods, especially in the area of topology design, are reviewed in the introduction to the paper by Kita and Toyoda (2000). The work of Kita and Toyoda is the starting point of this review.

The objective of the topology design considered in is to find the optimal thickness distribution of a two-dimensional continuum (plate) under in-plane loads. The basic methodology presented by Kita and Toyoda consists of; 1: finite number of elements are identified as CA cells, 2: the cell neighborhood is identified as the elements sharing a common edge with the cell, for the rectangular FEM mesh used, this is a Moore-neighborhood, and 3: an update rule is devised, based on stresses in the neighborhood, to update the cell thickness.

This work contained some far-reaching features. They formulated the CA design rule, for the first time, as a local optimization problem at the cell (element) level. They based the local update rule on the value of stress resultants in the neighborhood. Moreover, they provided an approximate sensitivity analysis as the basis for selecting the cell (element) level objective function. The main drawback of their method is that they depend on the Evolutionary Structural Optimization (ESO) method developed by Xie and Steven (1997). In the ESO, the Von Mises stress is used as a measure to eliminate elements in the domain that are not contributing to the load carrying capacity of the structure. This method is essentially heuristic and was criticized for its lack of mathematical foundations and premature convergence to suboptimal designs in a number of publications. Another disadvantage of this CA algorithm is the large number of iterations (in excess of a thousand) required to reach a converged topology.

\section{Finite Element Method}

Two-dimensional stress and deformation analysis problems form an important class in engineering design. These problems are of plain stress or plain strain type. We develop the finite element formulation for these problems using three-node triangular elements. A three-node triangle in which the displacement is represented as a linear function of the coordinates is called a Constant Strain Triangle (CST). An element of this type is referred to as a CST element. The strain and therefore the stress in these elements are constant. Once the element stiffness is developed, the procedure for global stiffness, boundary condition consideration, and the solution process follow the steps introduced by Chandrupatla (2004). The simplicity of the CST element helps us in the development of steps involved in the two-dimensional finite element formulation.

The problem studied for the purpose of this paper is a special case of plain strain. Plain stress problems, including problems that can be three dimensional mode and simpler two-dimensional forms are considered (Bathe 1982). Moreover, domain discretization using three-node triangular elements has been conducted and this is done to investigate studies on the effect of domain discretization on the response of the problems. A new program (subroutine) is developed for the state of the mentioned three-node to perform finite element analysis. Plain eight-node routine which is written in FORTRAN and published by Zienkiewicz et al. (2005) is reformed to prepare that subroutine for three-node constant strain triangles.

\section{Optimization Problem Formulation}

\subsection{Single-Objective Optimization Problem}

Results reported by Arora (2004), show that the selection of design variables greatly influences the problem formulation. Once the problem is properly formulated, methods, schemes or algorithms could be applied to solve it. Arora proposed a five step procedure to formulate design optimization problems which is applicable for most optimization problems; project/problem statement, data and information collection, identification or definition of design variables, identification of objective function(s) and identification of constraints.

All optimization problems have at least one optimization criterion that could be used to compare different designs and determine an optimum solution. Most engineering design problems must also satisfy certain equality or inequality (or both) constraints. A standard form of the design optimization model for a Single-Objective Optimization Problem (SOOP) which complies with the literature is as follows:

Find an n-vector $X=\left(x_{1}, x_{2}, \ldots, x_{n}\right)$ of design variables to minimize (maximize) a cost (profit) function

$$
f(x)=f\left(x_{1}, x_{2}, \ldots, x_{n}\right)
$$


subject to $p$ equality constraints

$$
h_{j}(x)=h_{j}\left(x_{1}, x_{2}, \ldots, x_{n}\right)=0 ; j=1 \text { to } p
$$

and $l$ inequality constraints

$$
g_{i}(x)=g_{i}\left(x_{1}, x_{2}, \ldots, x_{n}\right) \leq 0 ; i=1 \text { tol }
$$

and also $q$ upper and lower limits on the design variables

$$
x_{k}^{(L)} \leq x_{k} \leq x_{k}^{(U)} ; k=1 \text { to q }
$$

\subsection{Multi-Objective Optimization Problem}

A Multi-Objective Optimization Problem (MOOP) has a number of objective functions which are to be minimized or maximized. As in the SOOP, here too the problem usually has a number of constraints which any feasible solution (including the optimum solution) must satisfy. MOOP in its standard form as mentioned in the literature by Arora (2004) or Deb (2002) is stated as follows:

Find an (or set of) n-vector $X=\left(x_{1}, x_{2}, \ldots, x_{n}\right)$ of design variables to minimize (maximize) cost (profit) functions

$$
\begin{aligned}
& F(x)=\left(f_{1}\left(x_{1}, x_{2}, \ldots, x_{n}\right), f_{2}\left(x_{1}, x_{2}, \ldots, x_{n}\right),\right. \\
& \left.\ldots, f_{m}\left(x_{1}, x_{2}, \ldots, x_{n}\right)\right) ; m=1 \text { to } M
\end{aligned}
$$

subject to the $p$ equality constraints

$$
h_{j}(x)=h_{j}\left(x_{1}, x_{2}, \ldots, x_{n}\right)=0 ; j=1 \text { to } p
$$

and the $l$ inequality constraints

$$
g_{i}(x)=g_{i}\left(x_{1}, x_{2}, \ldots, x_{n}\right) \leq 0 ; i=1 \text { tol }
$$

and also $q$ upper and lower limits on the design variables

$$
x_{k}^{(L)} \leq x_{k} \leq x_{k}^{(U)} ; k=1 \text { to } q
$$

A solution $\mathrm{x}$ that does not satisfy all of the constraints and bounds is called an infeasible solution. Vice-versa, if any solution satisfies all constraints and variable bounds, it is known as a feasible solution.

\subsection{Design Variables, Constraints and Objective Functions}

The thicknesses of cells are considered as the design (decision) variables. So, design variables could be wrote as a vector as follows:

$$
f(x)=f\left(x_{1}, x_{2}, \ldots, x_{n}\right)
$$

where $X_{i}=$ thickness of the updated cell $i$ and $n=$ the number of cells.

Additionally, to formulate the optimization problem for each element individually, as Kita and Toyoda introduced, a special constraint condition, called CA-constraint condition, is considered. This CA-constraint condition is defined so as to minimize the variation of the equivalent stress of the neighboring cells with respect to the variation of the thickness of the updated cell. 
The first objective function of this optimization problem is to minimize the weight of the updated cells. Considering the material and the area of the cells as invariant parameters, the first objective function, which is an explicit function of the design variables, can be defined as follows:

$$
f_{1}=\left(x_{i} / t_{0}\right)^{2}
$$

In this equation $t_{0}$ is the initial thickness of the cell and $X_{i}=$ thickness of the updated cell $i$ and $n=$ the number of cells.

As implied in the previous part, the second objective function is to minimize the deviation between the yield stress of the material and the Von Mises equivalent stress at the cells. This aim is also expressed as follows:

$$
f_{2}=\left(\sigma_{0}-1\right)^{2}
$$

where $\sigma_{0}$ is the ratio of the Von Mises equivalent stress to the yield stress of the material.

This objective function is an implicit function of the design variables, so it is not possible to formulate the objective function explicitly in terms of the design variables alone. Instead, the intermediate variable, which is a type of stress ratio, is used to formulate the function.

In this paper, CA-constraint conditions are applied as defined by Kita and Toyoda. These conditions are explained as follows:

$$
g_{i}=\frac{\tilde{\sigma}_{i}}{\tilde{\sigma}_{i}^{0}}-1 \equiv \sigma_{i}-1=0,(i=1, \ldots, 3)
$$

where $\sigma_{i}$ denotes the ratio of equivalent stresses at the neighboring cell $i$ at the present step to the preceding step. Therefore, this equation ensures that the variation of the equivalent stress at the neighboring cell is small.

\subsection{Converting MOOP to SOOP}

A range of methods is available to convert the MOOP into a substitute problem with a scalar objective that can be solved with the tools of SOOP (Marler R. T. \& Arora J. S. 2004; Athan T. W. \& Papalambros P. Y., 1996). One of the most common general scalarization methods for MOOP is the global criterion method in which all objective functions are combined to form a single function. Linearly weighted sum method (WSM) is the most common approach to MOOP, which is a form of GCM. Applying WSM, after the objectives are normalized, a composite objective function $\mathrm{F}(\mathrm{x})$ can be formed by summing up the weighted normalized objectives and the MOOP given in equation (5) is then converted to a SOOP as follows:

$$
F(x)=\sum_{i=1}^{M} w_{i} f_{i}\left(x_{1}, x_{2}, \ldots, x_{n}\right)
$$

where, $\mathrm{w}$ is a non-zero positive vector of weights typically set by the decision maker such that $\sum_{i=1}^{M} w_{i}=1$.

If all of the weights are positive, the minimum of (13) is Pareto optimal (Zadeh 1963). In the other words, minimizing (13) is sufficient for Pareto optimality. Steuer (1989) mathematically relates the weights to the decision-makers preference function. Das and Dennis (1997) provide a graphical interpretation of the weighted sum method for two-objective problems to explain some of its deficiencies. Eschenauer et al. (1990) give a brief depiction of the method in criterion space. Koski and Silvennoinen (1987) discuss and illustrate the weighted sum method as a special case of methods that involve a p-norm.

Misinterpretation of the theoretical and practical meaning of the weights can make the process of intuitively selecting nonarbitrary weights an inefficient chore (Marler R. T. \& Arora J. S. 2004). Consequently, many authors have developed systematic approaches to selecting weights, surveys of which are provided by Eckenrode (1965), Hobbs (1980), Hwang and Yoon (1981), and Voogd (1983). Rao and Roy (1989) provide a method for determining weights based on fuzzy set theory. For cases in which the relative importance of the objective functions is unclear, Wierzbicki (1986) provides an algorithm that calculates weights based on the aspiration point and the utopia point.

Many authors touch on difficulties with the weighted sum method (Koski 1985; Stadler 1995; Athan and Papalambros 1996; Das and Dennis 1997; Messac et al. 2000a, b). Despite the many methods for determining weights, a satisfactory, a priori selection 
of weights does not necessarily guarantee that the final solution will be acceptable; one may have to resolve the problem with new weights. In fact, weights must be functions of the original objectives, not constants, in order for a weighted sum to mimic a preference function accurately (Messac 1996).

In this paper, using the weighted sum method, the new objective function as a linear combination of the two objective functions, mentioned in the previous section, is defined as follows:

$$
f_{3}=w_{1} f_{1}+w_{2} f_{2}
$$

here, $w_{1}$ and $w_{2}$ are defined as stated by Kita and Toyoda so that the following conditions are satisfied:

$$
\begin{aligned}
& w_{1}+w_{2}=1 \\
& w_{2}=\left\{\begin{array}{lll}
\sigma_{0} & \text { if } & \sigma_{0}<1 \\
1 & \text { if } & \sigma_{0} \geq 1
\end{array}\right\}
\end{aligned}
$$

The weight parameters refer to the relative importance of the objectives with regard to the amount of $\sigma_{0}$. To clear the weight vector some special cases are discussed below.

If $\sigma_{0} \geq 1$ then $w_{2}=1$ and $w_{1}=0$, so the composite objective function will be the minimization of the following function:

$$
f_{3}=f_{2}=\left(\sigma_{0}-1\right)^{2}
$$

In other words, in this case, the topology optimization is performed to minimize the variation of stresses during the optimization process.

On the other hand, for relatively small amounts of $\sigma_{0}$, the weight parameters would be $w_{2} \approx 0$ and $w_{1} \approx 1$. Hence, the objective function is formed as follows:

$$
f_{3} \approx f_{1}=\left(x_{i} / t_{0}\right)^{2}
$$

In this case the objective function of the optimization problem is weight minimization of cells.

Multiplying the penalty parameter $p$ into the CA-constraint condition and adding it to the objective function $f_{3}(x)$, the penalty function can be obtained as follows:

$$
\begin{aligned}
f= & w_{1} f_{1}+w_{2} f_{2}+p \sum_{i=1}^{3} g_{i}^{2}= \\
& w_{1}\left(x_{i} / t_{0}\right)^{2}+w_{2}\left(\sigma_{0}-1\right)^{2}+p \sum_{i=1}^{3}\left(\sigma_{i}-1\right)^{2}
\end{aligned}
$$

Using the Taylor's expansion, and other mathematical calculations, $\delta\left(x_{1} / t_{0}\right)$ can be obtained through the following formula:

$$
\delta\left(x_{1} / t_{0}\right)=\frac{\left[w_{1}\left(x_{1} / t_{0}\right)^{2}+w_{2}\left(\sigma_{0}-1\right) \sigma_{0}+p \sum_{i=1}^{3}\left(\sigma_{i}-1\right) \sigma_{i}\right]\left(x_{1} / t_{0}\right)}{w_{1}\left(x_{1} / t_{0}\right)^{2}+w_{2}\left(\sigma_{0}\right)^{2}+p \sum_{i=1}^{3}\left(\sigma_{i}\right)^{2}}
$$

During the updating of the thickness of cells, the following formula is used to decrease or increase the thickness:

$$
\left(x_{1} / t_{0}\right)^{k+1}=\left(x_{1} / t_{0}\right)^{k}+\delta\left(x_{1} / t_{0}\right)
$$

where the superscripts $k$ and $k+1$ represent the number of the iteration.

The termination criteria could be as follows: 


$$
f^{k+1}-f^{k}<\varepsilon
$$

where, $\varepsilon$ is the termination parameter which should be set in the initialization process. In this article to illustrate the results in long iterations, it is set to a very small amount.

\section{Execution Process}

Figure 3 display the flowchart which is used to implement the optimization procedure. The algorithm which is proposed by Sanaei ans Babaei (2010) is generalized and new features are considered in the procedure to create the design domain, divide the structure into small triangle elements, choosing the shape of elements to achieve better results. The stages of execution process are cited as follows:

Stage 1: Create the model (design domain), using the input data such as type of the structure (Deep or Simple Beam), structural dimensions, type of analysis, number of cells, material properties, design constraints, boundary conditions and loading information.

Stage 2: Structural analysis, to obtain stresses in elements using the prepared finite element method, square or triangular elements. The equivalent Von Mises stress at the cell $i$, is calculated as follows:

$$
\sigma_{\text {evon }}^{i}=\sum_{j=1}^{3} \alpha_{j} \cdot \sigma_{\text {von }}^{j}
$$

where $\sigma_{\text {evon }}^{i}$ denotes the equivalent Von Mises stress at the cell $i$; $\sigma_{v o n}^{j}$ is the Von Mises stresses at the neighboring cell $i$; and $\alpha_{j}$ is an adjustable weight parameter to consider stress at each neighbor in calculation of equivalent stresses.

Von Mises stresses at the cell $i$, is obtained as:

$$
\sigma_{v o n}^{i}=\sqrt{\sigma_{1}^{2}-\sigma_{1} \cdot \sigma_{2}+\sigma_{2}^{2}}
$$

where $\sigma_{v o n}^{i}$ denotes the Von Mises stress at the cell $i ; \sigma_{1}, \sigma_{2}$ denote the stresses at principle axes.

In this research a same value of $1 / 3$ is employed for weight parameters $\alpha_{j}$ to calculate the equivalent Von Mises stresses at each cell.

Stage 3: Check the termination criteria using Eq. 22. If the difference of objective functions between two consecutive stages of Eq. 22 is less than the $\varepsilon$, the termination criteria is met, so stop. Otherwise, go to the next stage.

Stage 4: Design variables update, with respect to stress distribution and updating rule using Eq. 21.

Stage 5: Modify the topology of the structure by deleting the cell elements which have small thickness. Go to stage 2.

\section{Numerical Case Studies and Results}

Laterally stable steel beams can fail only by flexure, shear or bearing, assuming the local buckling of slender components does not occur. These three conditions are the criteria for limit state design of steel beams. It is generally accepted in the literature that when the length to depth ratio of a beam is smaller than 5 then the behavior of the beam is shear and shear stresses will control the safety of the beam. On the other hand, if a beam is long in length and slim in the cross sectional area then flexural behavior of the beam will govern the safety. In this section, the developed algorithm is applied to two different types of beams with flexural and shear behavior to demonstrate the efficiency and accuracy of the presented scheme. For the proposed algorithm the penalty parameter is set to 10. The optimized topology of these case studies is obtained after repeating the optimization process. The algorithm is coded in FORTRAN and structures are analyzed using the developed Constant Strain Triangle finite element method. 


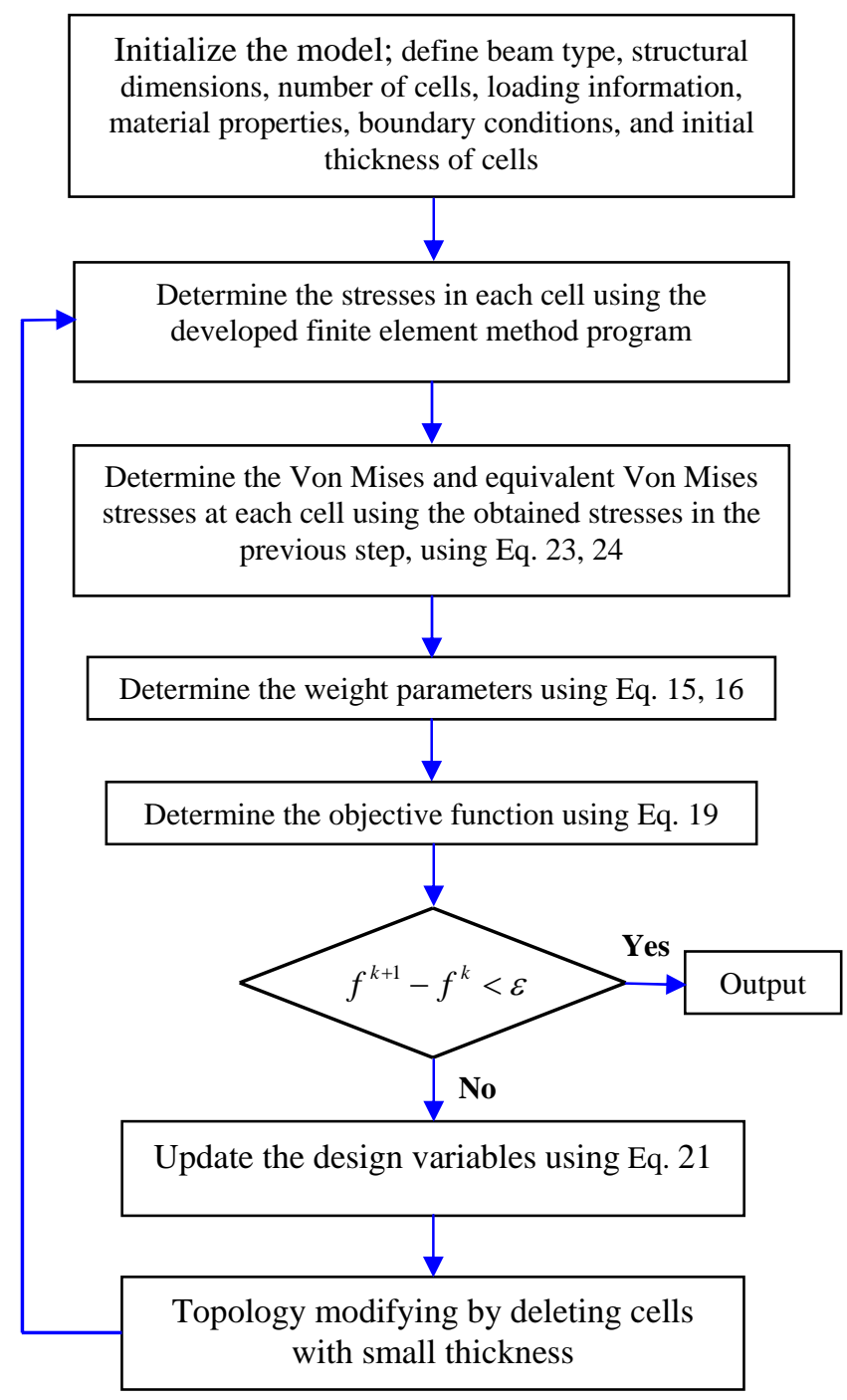

Figure 3. The flowchart of execution process

\subsection{Deep Beam}

The cantilever deep beam shown in Figure 4 shows the design domain, boundary conditions, and joint numbering for point loading. The material density is $490 \mathrm{lb} / \mathrm{ft}^{3}\left(7850 \mathrm{kgf} / \mathrm{m}^{3}\right)$ and the modulus of elasticity is $29 \mathrm{ksi}$ (200 MPa). The Poisson's ratio is 0.2. The design domain is divided into 768 triangle cells (48 in horizontal, 16 in vertical). The elements are subject to the stress limit of $\sigma_{c}=0.8 \times \sigma_{0}$, which $\sigma_{0}$ is the maximum stress at the initial profile. The thickness of each element is considered as a design variable, so in total there are 768 design variables. The initial thickness value for the elements is set to equal 40 in (1.016 $\mathrm{m})$. In this example three cases are considered. Table 1 illustrates the loading conditions for each case.

Table 1. Loading conditions for the deep beam

\begin{tabular}{cccc} 
& Case 1 & Case 2 & Case 3 \\
\cline { 2 - 4 } Load & Value $(\mathrm{N})$ & Value $(\mathrm{N})$ & Value $(\mathrm{N})$ \\
\hline P1 & 20 & 0 & 20 \\
P2 & 0 & 20 & 20 \\
\hline
\end{tabular}


The behavior of the deep beam is more in shear than flexural. Figure 5 (a, b, and c) displays the optimized topology for the three mentioned cases, obtained using the developed algorithm. The illustrated thickness distributions are obtained after 400 iterations. Figure 6 demonstrate the final topology for this beam after 1500 iterations, reported by Kita et al. These results show that the mentioned scheme in this paper achieves the optimum topology in less iteration, which illustrates the accuracy and efficiency of the scheme developed in this research.

Case 1: The convergence histories of the mean and the maximum stresses and the total weight of the structures with respect to the number of iterations are demonstrated in figure 7(a). Figure 7(b) shows the convergence histories of the same case, reported by Kita and Toyoda. The maximum stress, $\sigma_{\max }$, decreases rapidly when 300 iterations and then converges asymptotically to the reference stress $\sigma_{c}=0.8 \times \sigma_{0}$, while in figure 7(b) $\sigma_{\max }$ decreases rapidly when 600 iterations and then converges asymptotically to the reference stress. The mean stress, $\sigma_{m}$, increases rapidly when 150 iterations and then increases gradually to the reference stress, while in 7(b) $\sigma_{m}$ increases gradually to the reference stress. Both figures, (7(a, b)), indicate that the stress distribution on the whole structure tends to become uniform. Finally, the total weight of the structure decreases rapidly when 150 iterations and then decreases gradually to about $35 \%$ of its initial weight, while in 7 (b) decreases to $40 \%$.

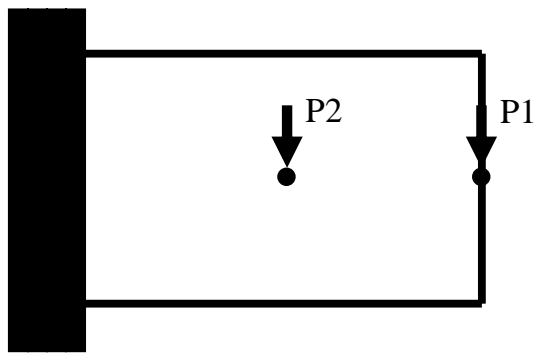

Figure 4. Design domain and boundary conditions for the deep beam

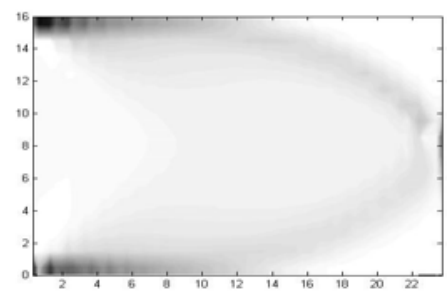

(a): Optimized topology for deep beam after 400 iterations (case 1)

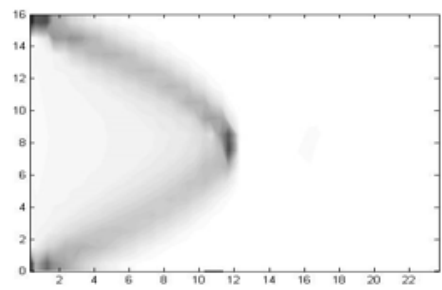

(b): Optimized topology for deep beam after 400 iterations (case 2)

Figure 5. Thickness distribution for topology optimization of deep beam 


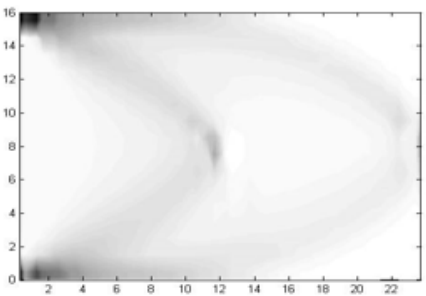

(c): Optimized topology for deep beam after 400 iterations (case 3)

Figure 5. Thickness distribution for topology optimization of deep beam (continued)

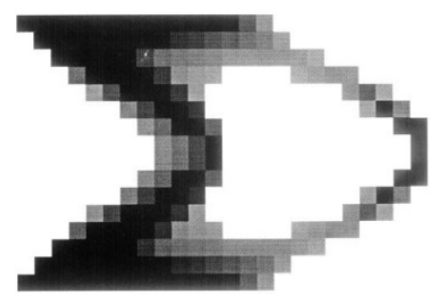

Figure 6. Thickness distribution at the final profile (case 3) $\left(1500^{\text {th }}\right.$ iteration) reported by Kita and Toyoda

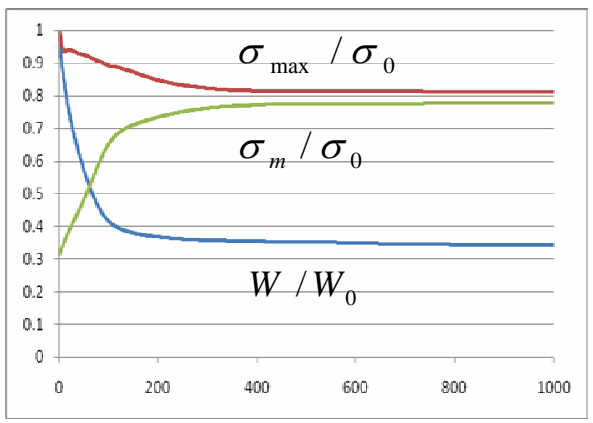

Number of Iterations

(a): Triangle cells

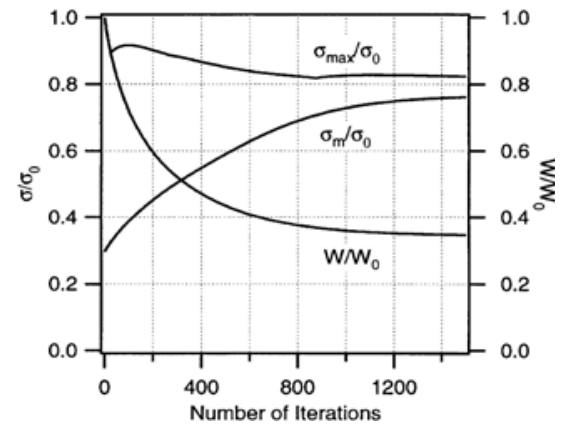

(b): Quadrangle cells (Kita and Toyoda 2000)

Figure 7. Convergence histories of maximum and mean stresses and weight for case 1

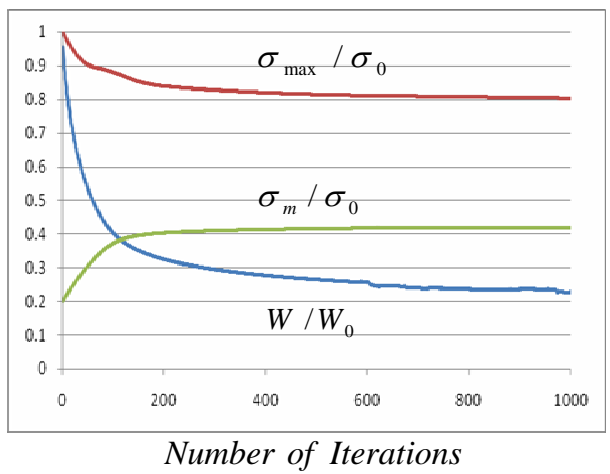

(a): Triangle cells

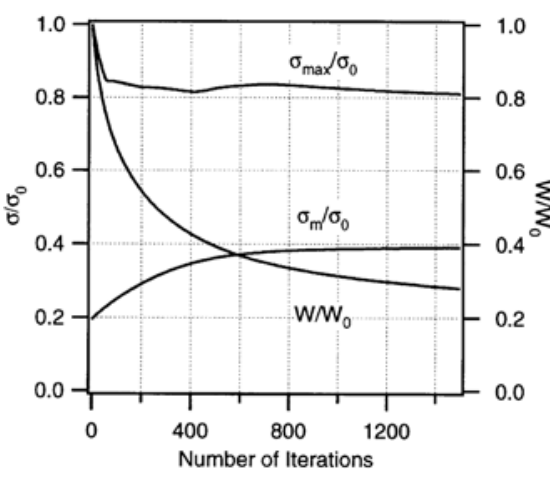

(b): Quadrangle cells (Kita and Toyoda 2000)

Figure 8. Convergence histories of maximum and mean stresses and weight for case 2 


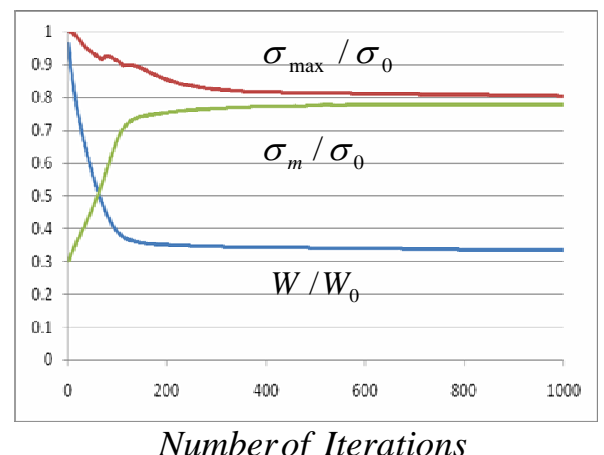

(a): Triangle cells

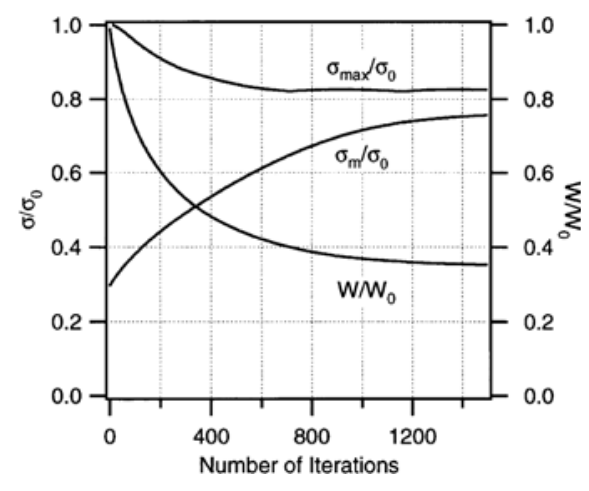

(b): Quadrangle cells (Kita and Toyoda 2000)

Figure 9. Convergence histories of maximum and mean stresses and weight for case 3

Case 2: The convergence histories of the mean and the maximum stresses and the total weight of the structures are demonstrated in figure 8(a), this research result, and in figure 8(b), reported by Kita and Toyoda. The maximum stress, $\sigma_{\text {max }}$, decreases rapidly when 200 iterations and then converges asymptotically to the reference stress $\sigma_{c}=0.8 \times \sigma_{0}$, while in figure 8(b) $\sigma_{\max }$ decreases rapidly when 600 iterations and then converges asymptotically to the reference stress. The mean stress, $\sigma_{m}$, increases rapidly when 150 iterations and then converges to more than half of the reference stress $(0.42)$, while in 8(b) $\sigma_{m}$ increases gradually and then converges to half of the reference stress (0.40). The mean stress in figure 7 (a) is greater than that in figure 7(b) (i.e. the stresses in triangle cells are higher than quadrangular cell's). On the other hand, the total weight in figure 7(a) is less than that in figure 7 (b). Both figures, 7(a, b), indicate that the stress distribution on the whole structure varies more than in case 1 . The total weight decreases gradually and the final structure is much lighter than that found in case 1 , about $23 \%$ of the initial weight, while in figure 8(b) decreases gradually to $30 \%$. This is because the load point is specified in the center of the structure and therefore the area of the structure which can be deleted is wider than that of case 1.

Case 3: The convergence histories of the mean and the maximum stresses and the total weight of the structures are demonstrated in figure 9(a). The maximum stress, $\sigma_{\max }$, decreases rapidly when 150 iterations and then converges asymptotically to the reference stress $\sigma_{c}=0.8 \times \sigma_{0}$, while in figure 9(b) $\sigma_{\max }$ decreases rapidly when 600 iterations and then converges to the reference stress. The mean stress, $\sigma_{m}$, increases rapidly when 150 iterations and then converges to the reference stress, but in figure 9(b) $\sigma_{m}$ increases gradually and then converges to the reference stress. The stress distribution become almost uniform in both figures $9(\mathrm{a}, \mathrm{b})$, and it is similar to case 1 and different from case 2 . The total weight of the structure decreases rapidly when 150 iterations and then converges to 33\% of the initial weight, while in figure 9(b) decreases gradually to 35\% of the initial weight of the structure. The final profile, as illustrated in figure 5(c), is very similar to that found by superposing the final profiles of case 1 and case 2, as shown in figures 5(a) and 5(b), respectively.

\subsection{Flexural Beam}

Figure 10 shows the design domain and the boundary conditions for a one-bay simply connected beam. The structural behavior of this beam is more flexural than shear. Joint numbering for point and distributed loading is demonstrated in Figure 10 . The material density is $490 \mathrm{lb} / \mathrm{ft}^{3}\left(7850 \mathrm{kgf} / \mathrm{m}^{3}\right)$ and the modulus of elasticity is $29 \mathrm{ksi}$ (200 MPa). The Poisson's ratio is 0.2 . The design domain is divided into 192 triangle cells (48 in horizontal, 4 in vertical). The elements are subject to a stress limit of $\sigma_{c}=0.8 \times \sigma_{0}$, which $\sigma_{0}$ is the maximum stress at the initial profile. The thickness of each element is considered as a design variable, so in total there are 192 design variable. The initial thickness value for the elements is set to equal 40 in (1.016 $\mathrm{m}$ ). In this example three cases are investigated. Table 2 illustrates the loading conditions for each case, including point and distributed loading. 
Table 2. Loading conditions for the simple beam

\begin{tabular}{cccc} 
& Case 1 & Case 2 & Case 3 \\
\cline { 2 - 4 } Load & Value $(\mathrm{N})$ & Value $(\mathrm{N})$ & Value $(\mathrm{N})$ \\
\hline P1 & 20 & 0 & 0 \\
P2 & 0 & 20 & 0 \\
P3 & 0 & 0 & 100 \\
\hline
\end{tabular}

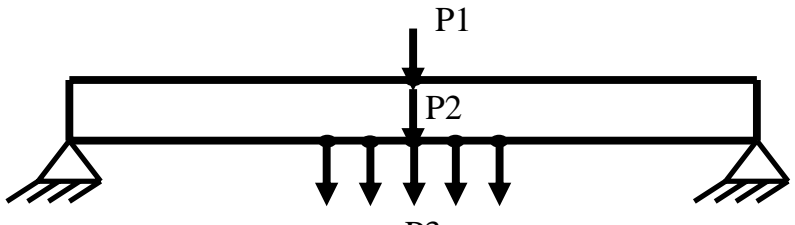

P3

Figure 10. Design domain and boundary conditions for the simply connected beam

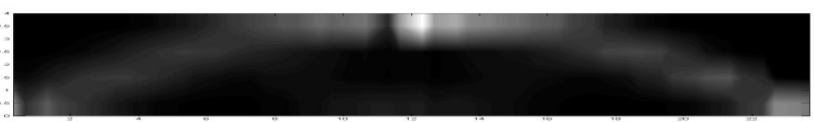

(a): Optimized topology for one-bay beam (case 1)

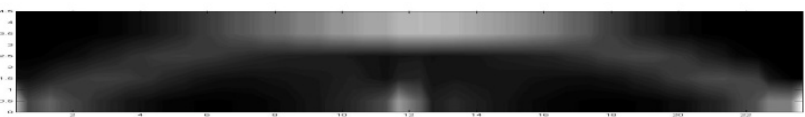

(b): Optimized topology for one-bay beam (case 2)

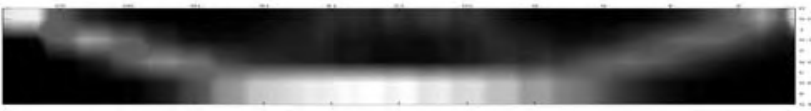

(c): Optimized topology for one-bay beam (case 3)

Figure 11. Thickness distribution for topology optimization of simply connected one-bay beam

Figure 11 (a, b, and c) displays the final topology for the three cases. Black cells are those deleted during the optimization process and white cells have thickness, relating to their brightness. The illustrated thickness distributions are obtained after 400 iterations. The convergence histories of the stresses (max and mean) and the total weight of the structures are shown in figures 12 , 13, and 14. In all cases, the maximum stress, $\sigma_{\max }$, decreases rapidly when 200 iterations and then converges asymptotically to the reference stress $\sigma_{c}=0.8 \times \sigma_{0}$. The mean stress, $\sigma_{m}$, increases rapidly when 300 iterations and then converges to near the reference stress. The total weight of the beams decreases rapidly when 300 iterations and then converges to about $38 \%$ of the initial weight. 


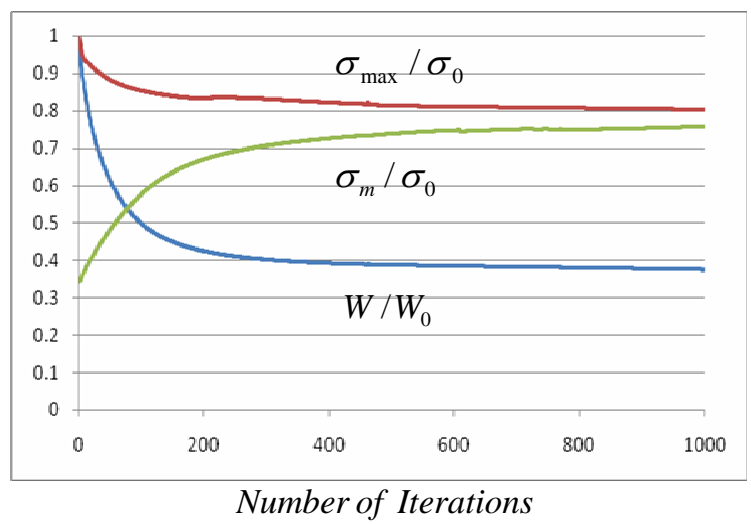

Figure 12. Convergence histories of maximum and mean stresses and weight for case 1

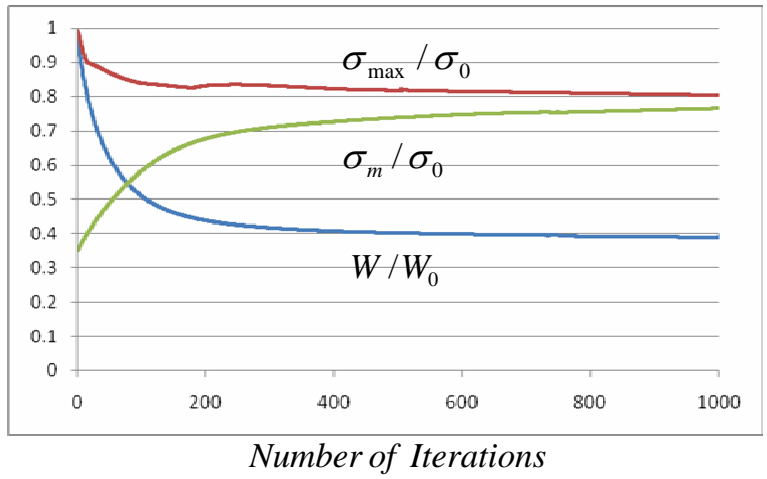

Figure 13. Convergence histories of maximum and mean stresses and weight for case 2

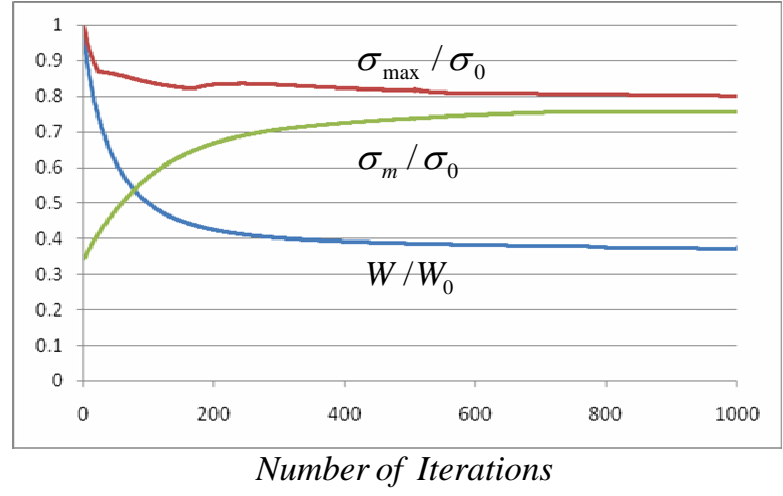

Figure 14. Convergence histories of maximum and mean stresses and weight for case 3

\section{Conclusions and Remark}

In this paper a topology optimization method is presented for optimization of structures on which the concept of CA has been applied. The method is applied for topology optimization of two-dimensional elastic structures and the design domain is divided into triangles in order to perform finite elements analysis, which is developed using FORTRAN. One of the important features of CA is defining the neighboring for cells, so that it will affect the convergence and final result of the problem. In this article, a new neighborhood for triangular elements is proposed and the structures are analyzed by CST finite element method. The algorithm is also developed to continuum structures, with shear and flexural behavior. The final profiles of the structures, which are obtained using the proposed scheme in this paper, are similar to the results reported in existing studies based on CA concept (Kita and 
Toyoda 2000; Kundu et al. 1997a, b; Inou et al. 1994, 1998) and using the gradient search scheme concept (Suzuki and Kikuchi 1991; Bendsoe and Kikuchi 1988).

The convergence histories of maximum and mean stresses and weight indicate the efficiency and accuracy of solutions obtained for optimized topology of the structures. In the other words, by the developed scheme in this paper the final topologies for the case studies are obtained in less iteration. Optimum topology for the deep beam in Cases 3, obtained after 400 iterations, is compared with layout obtained by Kita and Toyoda, which was obtained after 1500 iterations. The following modifications are the main reasons for rapid convergence: a) since the triangle elements are employed, the quadrangle cells are divided into two triangle elements. So, the number of elements (cells) is increased. Obviously, it will affect the results such as the weight of the structure at the final step, when applying cells updating in CA. In the other word, in the updating process, cells are affected by smaller neighbors; b) the new neighborhood for cells, which is for triangle elements, is proposed and considered in the case studies; c) the cells are deleted from the boundary of the structure to the centre. This rule is applied from the first iteration for all cells with small Von Mises stress; d) the nature and characteristic of triangle elements in FEM analysis, comparing quadrangular elements, affects the analysis process. Based on the results of the three case studies for deep beam and in comparison to those reported in the literature, it can be proposed that the method developed in this paper is valid and can be applied to other structures. As a future research study, one can develop this algorithm to truss and frame structures.

\section{Nomenclature}

CA Cellular Automata

CST Constant Strain Triangle

ESO Evolutionary Structural Optimization

MOOP Multi-Objective Optimization Problem

SOOP Single-Objective Optimization Problem

WSM Weighted Sum Method

GCM Global Criterion Method

\section{References}

Arora J. S. (2004), “Introduction to Optimum Design, McGraw-Hill Book Comp., 2nd Ed

Athan T. W., Papalambros P. Y., (1996), "A Note on Weighted Criteria Methods for Compromise Solution Multi-Objective Optimization” Engineering Optimization, Vol. 27, pp. 155-176

Bathe K.-J. (1982), “Finite Element Procedures in Engineering Analysis” Prentice-Hall

Bendsoe M.P., Kikuchi N., (1988) “Generating Optimal Topologies in Structural Design using a Homogenization Method” Comp. Meth. Appl. Mech. \& Eng. 71, 197-224

Chandrupatla T. (2004), "Finite Element Analysis for Engineering and Technology”, University Press

Das I., Dennis J. E. (1997) "A Closer Look at Drawbacks of Minimizing Weighted Sums of Objectives for Pareto Set Generation in Multicriteria Optimization Problems” Struct. Optim. 14, 63-69

Deb K. (2002), Multi-Objective Optimiz-ation Using Evolutionary Algorithms, John Wiley

Eckenrode R. T. (1965) “Weighting Multiple Criteria” Manage. Sci. 12, 180-1

Eschenauer H., Koski J., Osyczka A. (eds.), (1990) “Multicriteria Design Optimization Procedures and Applications” Berlin: Springer-Verlag

Hobbs B. F. (1980) “A Comparison of Weighting Methods in Power Plant Siting” Decis. Sci. 11, 725-737

Hwang C.-L., Yoon K., (1981) "Multiple Attribute Decision Making Methods and Applications” a state-of-the-art survey. In: Beckmann, M.; Kunzi, H.P. (eds.) Lecture Notes in Economics and Mathematical Systems, No. 186. Berlin: Springer-Verlag

Kita E., Toyoda T. (2000), "Structural Design Using Cellular Automata” Structural Multidisciplinary Optimization, 19, 64-73, Springer-Verlag

Koski J., (1985) "Defectiveness of Weighting Method in Multicriterion Optimization of Structures" Commun. Appl. Numer. Methods 1, 333-337

Koski J., Silvennoinen R. (1987) “Norm Methods and Partial Weighting in Multicriterion Optimization of Structures, Int. J. Numer. Methods Eng. 24, 1101-1121

Kundu S., Oda J., Koishi T., (1997a) "A Self-Organizing Approach to Optimization of Structural Plates using Cellular Automata” In: Gutkowski W., Mroz Z. (eds.), Proc. WCSMO-2, Second World Congress on Structural and Multidisciplinary Optimization (held in Zakopane, Poland), pp. 173-180. Warsaw: Polish Academy of Science

Kundu S., Oda J., Koishi T., (1997b) “Design Computation of Discrete Systems using Evolutionary Learning” In: Gutkowski W., Mroz Z. (eds.), Proc.WCSMO-2, Second World Congress on Structural and Multidisciplinary Optimization (held in Zakopane, Poland), pp. 173-180. Warsaw: Polish Academy of Science

Inou N., Shimotai N., Uesugi T. (1994), “A Cellular Automaton Generating Topological Structures” In: McDonach, A.; Gardiner, P.T.; McEwan, R.S.; Culshaw, B. (eds.) Proc. 2nd European Conf. on Smart Structures and Materials 2361, pp. 47-50 
Inou N., Uesugi T., Iwasaki A., Ujihashi S. (1998), "Self Organization of Mechanical Structure by Cellular Automata” In: Tong P., Zhang T.Y., Kim J., (eds.) Fracture and Strength of Solids. Part 2: Behaviour of Materials and Structure (Proc. 3rd Int. Conf., held in Hong Kong, 1997), pp. 1115-1120

Marler R. T., Arora J. S. (2004), “Survey of Multi-Objective Optimization Methods for Engineering”, Struct Multidisc Optim 26, 369-395

Messac A., Sukam C. P., Melachrinoudis E. (2000a) “Aggregate Objective Functions and Pareto Frontiers: required relationships and practical implications” Optim. Eng. 1, 171-188

Messac A., Sundararaj G. J., Tappeta R. V., Renaud J. E., (2000b) "Ability of Objective Functions to Generate Points on Nonconvex Pareto Frontiers” AIAA J. 38, 1084-1091

Messac A., (1996) "Physical Programming: Effective Optimization for Computational Design” AIAA J. 34, 149-158

Rao J. R., Roy N., (1989) "Fuzzy Set Theoretic Approach of Assigning Weights to Objectives in Multicriteria Decision Making” Int. J. Syst. Sci. 20, 1381-1386

Sanaei E, Babaei M. (2010), Topology Optimization of Structures using Cellular Automata with Constant Strain Triangles, Personal Communication with the authors.

Stadler W. (1995), “Caveats and Boons of Multi-criteria Optimization” Microcomput. Civ. Eng., 10, 291-299

Steuer, R.E. (1989), "Multiple Criteria Optimization: Theory, Computation, and Application”, Malabar: Robert E. Krieger Publishing

Suzuki K., Kikuchi N., (1991), “Shape and Topology Optimization using the Homogenization Method” Comp. Meth. Appl. Mech. \& Eng. 93, 291-318

Ulam S. (1952), “Random Processes and Transformations” Proceedings of the Int. Congress of Mathematics, Vol. 2, pp. 85-87

Voogd H. (1983) “Multicriteria Evaluation for Urban and Regional Planning” London: Pion

Von Neumann J. (1966), "Theory of Self-Reproducing Automata” University of Illinois Press

Wierzbicki A. P., (1986), "A Methodological Approach to Comparing Parametric Characterizations of Efficient Solutions. In: Fandel G., Grauer M., Kurzhanski A., Wierzbicki A. P. (eds.), "Large-Scale Modeling and Interactive Decision Analysis", Lecture Notes in Economics and Mathematical Systems, No. 273, pp. 27-45. Berlin: Springer-Verlag

Xie Y. M., Steven G. P. (1997), "Evolutionary Structural Optimization” Springer-Verlag, Berlin

Xie Y. M., Steven G.P. (1993), “A simple Evolutionary Procedure for Structural Optimization” Computers \& Structures, No. 49, 885-896

Xie Y. M., Steven G.P. (1994), “Optimal Design of Multiple Load Case Structures Using an Evolutionary Procedure” Eng. Comput., 11, 295-302

Zadeh, L. A. (1963), “Optimality and Non-Scalar-Valued Performance Criteria”, IEEE Trans. Autom. Control AC-8, 59-60

Zienkiewicz O. C., Taylor R.L. (2005), “The Finite Element Method for Solid and Structural Mechanics”, Elsevier ButterworthHeinemann, 6th Edition

\section{Biographical Notes}

E. Sanaei is of Department of Civil Engineering, Iran University of Science and Technology, Iran

M. Babaei is a scholar in the E. Sanaei is of Department of Civil Engineering, Iran University of Science and Technology, Iran.

Received October 2010

Accepted April 2011

Final acceptance in revised form April 2011 